\title{
Problems and Solutions of Baghouse in Power Plants
}

\author{
Guozhu Weng \\ Department of Power Engineering, North China Electric Power University, Baoding, China
}

Keywords: power plants, baghouse, problems, solutions.

\begin{abstract}
Along with the rapid development of industry, the dust emitted by the factory is inevitable and baghouse has also been widely used in the power plants. This paper takes power plants as examples to elaborate on current problems of baghouse, such as frequent and easy damage on bags, smolder of dust and ash, serious dust container abrasion and so on. Some solutions such as improving power plants production and strengthening the quality of bags are put forward.
\end{abstract}

\section{Introduction}

In the era of heavy chemical industry, the economy is booming in China, but also has brought a series of serious problem to the environment. Iron and steel, cement, chemical industry, electric power develops rapidly, leading to further deterioration of the environment in local area. Baghouse can protect the environment against pollution with the dust removal efficiency of $99.99 \%$, therefore it is widely used in various industries in China. Dust and ash collected by baghouse can be recycled as effective resources, so the use of baghouse not only improve the environment, but also make economic benefit. However, at present there are still many problems of baghouse.

\section{Introduction of Baghouse}

\subsection{Introduction}

Baghouse is an efficient dust removal device. With environmental degradation as the side effects brought about by the economic growth in recent years, the output of baghouse is more and more and its variety is more and more extensive. In power plants, the dust is very much, and often the temperature is relatively high, so electrostatic precipitator technology is more used. However, the technology is limited by the dust properties, and the cyclone dust removal technology is not effective. We choose the baghouse because of its strong adaptability. The baghouse uses a mesh to separate solid particles from the air. According to the network used for filtration, it can be divided into fiber filtration, membrane filtration and particle filtration. Although the principles of these methods are different, but they can achieve the desired results. In power plants, the baghouse is a combination of fiber filtration, membrane filtration, and particle filtration. The mechanism of the dust collector is to intercept and screen the particles first through the fiber network. Then, it uses gravity and electrostatic effects to separate and achieve the purpose of purifying dust [1].

\subsection{Composition and Classification of Baghouse}

The dust cleaning device of a baghouse consists of a pulse valve, blowpipes, a gas storage bag, an inducer, filters and a control device. The two ends of the pulse valve are respectively connected with compressed air and blowpipes. Blowpipes blows several times to clean the dust. The blowing system and the compression piping system include the air tank, the blow pipes and other associated attachments. The air tank is a device that provides compressed air for storage and has a certain strength. First, the electrical signal is input so that the diaphragm is on standby. The air pressure makes diaphragm vibration to achieve a closed state. Compressed air must be dry and without oily substances, otherwise it will cause some damage to the baghouse.

The dust cleaning device of baghouse is an important component. According to the different cleaning methods, baghouse can be divided into the following forms: Mechanical-Shaker type, Reverse-Air type, Reverse-Jet type, sonic wave type and composite type. Among them, Mechanical-Shaker type uses the periodic operation of the machine to shock the filter bags, so as to 
achieve the purpose of cleaning. This method can also be divided into two types, namely, continuous shock and intermittent shock. The selection between the two types are based on actual conditions and the density of dust. Reverse-Jet type uses the compressed air instead of the machinery to hit the filter bags, and its principle is similar to the Mechanical-Shaker type 's, but shock way is different. When dust is deposited, pulse cleaning can be selected, and at the time of releasing compressed air, it will affect the atmospheric pressure of surrounding air. The main forms of baghouse are shown in Fig. 1, Fig. 2 and Fig. 3.

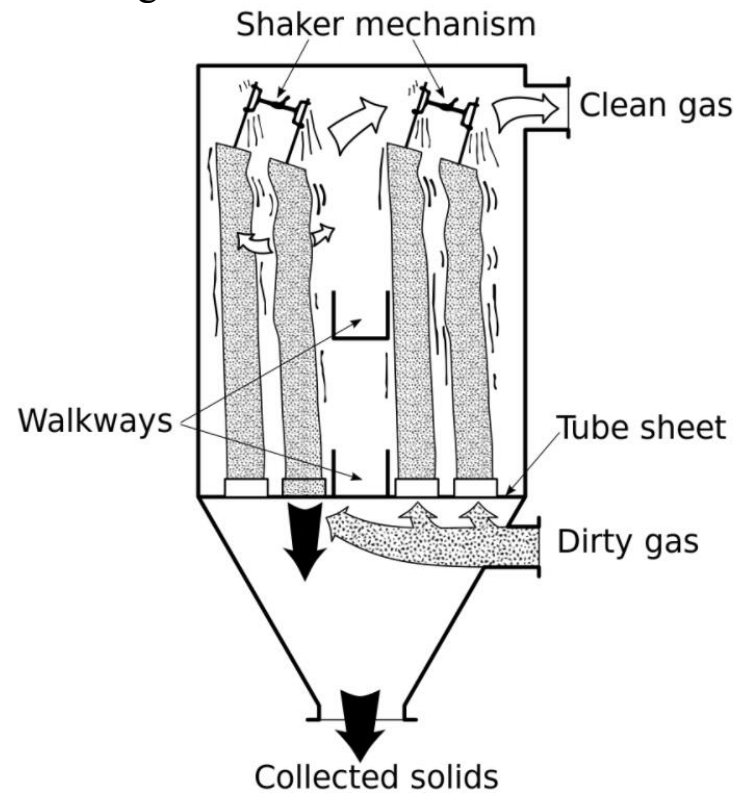

Fig. 1 Mechanical-Shaker type baghouse

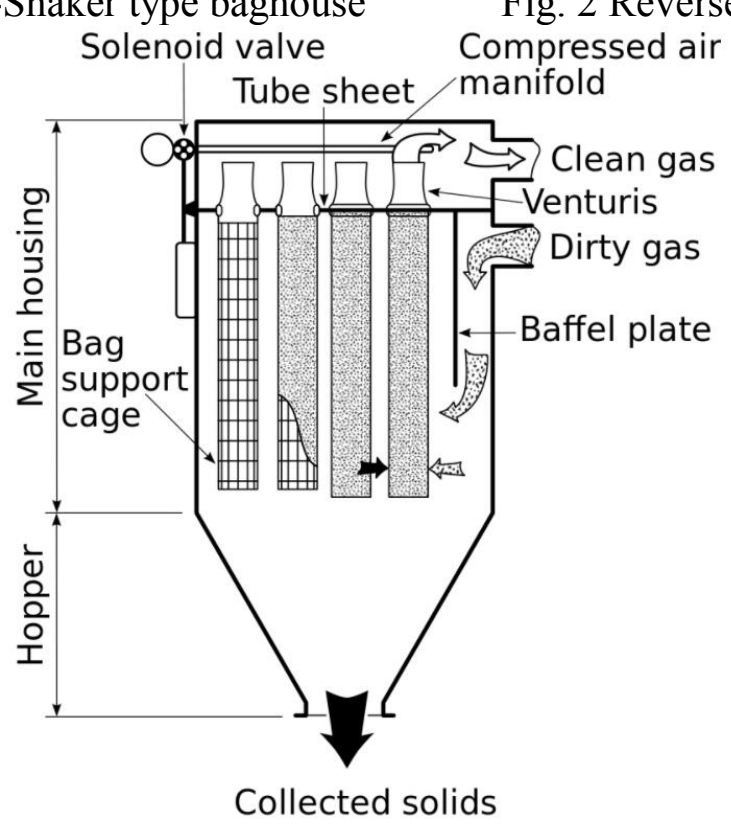

Fig. 3 Reverse-Jet type baghouse

\section{Research Status, Development and Trends in China}

As the modernization started late in China, there is no great progress in the baghouse. Initially, China introduced technology from the Soviet Union in the 50s of last century. Later, people make unremitting efforts and research institutes began to study, gradually imitating foreign advanced products. Since it got into the 90 's, people have made a breakthrough progress and baghouse has developed rapidly. In the recent period, with the rapid growth of the economy, the technology and equipment of baghouse are developing rapidly. Being more practical, it has related applications in all fields in China. At present, with the urgent need to protect the environment, both large factories and 
small workshops have used baghouse, which effectively reduces air pollution and plays a decisive role in the clean air. In the design process of baghouse, the space which dust need, dust density and other parameters should be calculated reasonably. Reasonable selection of parameters in the design can make the equipment work better. However, with the development of baghouse, there are many problems exposed.

\section{Problems Existing in Operation of Baghouse in Power Plants}

\subsection{Poor Running Efficiency and Short Bags Life}

The low efficiency is one of the most important problems in operation of baghouse. During the operation of the baghouse, excessive dust density will increase the load. It continues to run, resulting in damage to the equipment and even irreversible damage. Adopting large equipment will lead to high product cost, seriously affects the economic benefits. This is not beneficial to dust removal and resource utilization. Even though the power plant is running normally, the bag has a very short life. Wear occurs during use, especially at the opening and bottom of the filter bag. This phenomenon is caused by granular dust that rubs the filter bag when it is filtered. The maintenance cost is very high. Once the filter bag is damaged, dust enters the atmosphere immediately, causing great loss [2].

\subsection{Serious Dust Container Abrasion and Susceptible Smolder of Dust}

Under normal working conditions, the baghouse will be discharged after a certain time to clean the space and carry out dust removal again. Excessive dust density increases discharge rate. Dust container will be wear off in this process, therefore it needs to be replaced on time. But replacement costs are extremely high because dust is deposited in the dust collector. In the case of sufficient oxygen, it produces a great deal of heat due to friction. This will lead to smolder and baghouse breakdown, resulting in serious damage. Not only the economic benefit will be affected, but also production efficiency will decline.

\subsection{Susceptible Blocking in Key Parts}

The dust deposit makes it possible to clog up some small pipes in the baghouse. It can cause blockage in the channel and affect production. If clogging dust is not timely cleaned, it will seriously affect the work of equipment, leading to bad consequences [3].

\section{Cause Analysis and Solutions of Problems in Baghouse}

\subsection{Cause Analysis of Problems}

Fly dust contains many solid particles, which causes the filter bag and the dust container easy to wear, especially when the dust container is discharged. Pulsed jet frequency is too large, resulting in increased costs, causing unnecessary friction, resulting in equipment wear and tear [4]. In addition, there are a lot of combustible material in the dust. Containing a lot of heat, the dust deposit can come into being smolder, making bags worn and dirty gas poured into the atmosphere. The poor tightness of some bags is one of reasons for smolder, which causes good ventilation condition and help to combust [5]. Furthermore, it also makes dust flow inside and outside the filter bag and clog up some small pipes. Repetition causes vicious cycles.

\subsection{Solutions of Problems in Baghouse}

Transforming the equipment and modifying power generation mode can reduce dust production. Meanwhile, utilities should improve the production efficiency of the power plants and make regular equipment inspection, maintenance and cleaning, to ensure the normal operation of equipment. It is also an effective way to fundamentally control dust. Improving the production status of power plants, reducing the carbon content of dust, and making full use of carbon are also effective measures. The worn filter bags should be regularly checked and changed, and small pipes in the baghouse should keep in good ventilation by air flow regularly. Air flow should have high rate, so that the pipes are not blocked and the dust is in circulation. 


\section{References}

[1]. Zhou Shijia, Zeng Jianqin, Application of Baghouse in power Plant Boiler [J]. Energy Environmental Protection, 2017, (02) :5-7.

[2]. Wang lei, Fu qiwen, Lu zefeng, Reliability Analysis of Bag Dust Collector in Thermal Power Plant [J]. Huadian Technology, 2010, (05) :1-3.

[3]. Zhao Kunlun, Problems and Solutions in Pulsed Dust Removal of Filter Bag [J]. Technology and Engineering Application, 2009, (07) :25-30.

[4]. Zhang Lei, Shi Weibin, Cause Analysis of Bag Failure of Bag Filters for 350MW Turbine Boiler and Prevention [J]. Metallurgical Power, 2017, (04) :10-13.

[5]. Li Xiao, On the Factors Influencing Performance of Bag Dust Collector and Prevention Measures [J]. Gansu Metallurgy, 2016, (06) :125-126. 